\title{
Atenção primária à saúde nos 25 anos da Revista Ciência \& Saúde Coletiva
}

\author{
Primary health care in the 25 years of Journal Ciência \& Saúde \\ Coletiva
}

\author{
Aylene Bousquat (https://orcid.org/0000-0003-2701-1570) ${ }^{1}$ \\ Maria Guadalupe Medina (https://orcid.org/0000-0001-7283-2947) ${ }^{2}$ \\ Maria Helena Magalhães de Mendonça (https://orcid.org/0000-0002-3917-9103) ${ }^{3}$ \\ Patty Fidelis de Almeida (https://orcid.org/0000-0003-1676-3574) ${ }^{4}$ \\ Rosana Aquino (https://orcid.org/0000-0003-3906-5170) ${ }^{2}$ \\ Alaneir de Fátima dos Santos (https://orcid.org/0000-0002-7674-0449) ${ }^{5}$ \\ Ligia Giovanella (https://orcid.org/0000-0002-6522-545X) ${ }^{3}$
}

${ }^{1}$ Faculdade Saúde Pública, Universidade de São

Paulo. Av. Dr. Arnaldo 715, Cerqueira Cesar. 01246904 São Paulo SP Brasil. aylenebousquat@usp.br

${ }^{2}$ Instituto de Saúde

Coletiva, Universidade Federal da Bahia. Salvador BA Brasil.

${ }^{3}$ Escola Nacional de Saúde Pública Sergio Arouca, Fiocruz. Rio de Janeiro RJ

Brasil.

${ }^{4}$ Instituto de Saúde Coletiva,

Universidade Federal

Fluminense. Niterói RJ

Brasil.

${ }^{5}$ Faculdade de Medicina,

Universidade Federal

de Minas Gerais. Belo

Horizonte MG Brasil.

\begin{abstract}
Primary Health Care (PHC) is an area of study that has improved remarkably in the last decades. In Brazil, this academic production is highly expressed in the field of Collective Health. This paper aims to analyze the PHC production published in the first 25 years of the "Journal Ciencia er Saúde Coletiva" (CerSC). A narrative review was carried out, with analysis of the themes, methods, scale of analysis, partnerships, and authorship. A total of 295 papers were published, which corresponds to $5.9 \%$ of the total publications. A growing trend in papers addressing PHC was observed. The studies were mostly empirical (78.6\%), with a qualitative approach (58.0\%) and were predominantly local or municipal. Studies on health professionals were more frequent. The three prevailing themes were the health care model, PHC performance or effectiveness, and the work process. The profile found dialogues with the rich and diverse experience of Brazilian PHC. However, the incorporation of broader analyses is still challenging. The published papers highlighted the debates and contributed to the reflection and dissemination of the experience of Brazilian PHC, which was and is central to the construction of the Brazilian Health System.
\end{abstract}

Key words Primary Health Care, Bibliometrics, Periodicals as topic
Resumo A Atenção Primária à Saúde (APS) é uma área de estudo que tem crescido nacional e internacionalmente. No Brasil, esta produção se expressa sobremaneira no campo da Saúde Coletiva. O objetivo do artigo é o de analisar a produção sobre APS divulgada nos primeiros 25 anos da Revista Ciência é Saúde Coletiva (CひSC). Foi realizada uma revisão narrativa, com análise dos temas, dos métodos, da escala de análise, das parcerias estabelecidas e da autoria. Foram publicados 295 artigos, o que corresponde a 5,9\% do total de publicações. Observou-se tendência de crescimento de artigos versando sobre a APS. Os estudos foram na sua maioria empíricos (78,6\%), com abordagem qualitativa $(58,0 \%)$ e com predominio de abrangência local ou municipal. Quanto à população estudada, foram mais frequentes os estudos realizados com profissionais de saúde. Os três temas que predominaram foram: o modelo assistencial, o desempenho ou efetividade da APS e o processo de trabalho. O perfil encontrado dialoga com a rica e diversa experiência da APS brasileira, no entanto permanece o desafio de incorporar análises mais amplas. Os artigos publicados evidenciaram os debates e contribuíram para a reflexão e a divulgação da experiência da APS brasileira, que foi e é central para a construção do Sistema Único de Saúde.

Palavras-chave Atenção primária à saúde, Bibliometria, Publicações periódicas como assunto 


\section{Introdução}

Os primeiros 25 anos da Revista Ciência \& Saúde Coletiva (C\&SC), editada pela Associação Brasileira de Saúde Coletiva (Abrasco), são contemporâneos ao processo de construção do Sistema Único de Saúde (SUS) no Brasil. As páginas da C\&SC refletiram muitos dos debates, disputas, indagações, conquistas e retrocessos deste período ${ }^{1}$.

Sem dúvida, a criação do SUS e sua implementação significaram importantes mudanças na distribuição de poder político e responsabilidades entre as esferas de governo (nacional, estadual e municipal), entre o Estado e a sociedade civil por meio de novos mecanismos de participação social e de importante processo de descentralização, com transferência de poder decisório e competências do nível nacional para os níveis subnacionais. Outro ponto central na construção do SUS foi a definição do modelo assistencial e o papel da Atenção Primária à Saúde (APS) neste ${ }^{2,3}$.

O desenho do SUS foi influenciado por diversas propostas de modelos assistenciais com distintas racionalidades e experiências precedentes de caráter local, que ao longo destas três primeiras décadas foram conformando novas abordagens na APS e modelos assistenciais sempre em disputa, impulsionada por políticas nacionais, inclusive com mudanças políticas, gerenciais e organizacionais relevantes ${ }^{3,4}$. As disputas do modelo assistencial no SUS permearam a importante expansão dos serviços de atenção primária e da Estratégia Saúde da Família (ESF) ao longo destes mais de 20 anos, fundadas por distintas concepções do direito à saúde e de organização das práticas e inserções profissionais: entre acesso universal e atenção focalizada, entre o pronto-atendimento e o cuidado integral, resposta às necessidades coletivas e individuais, demanda espontânea e demanda programada, entre outras ${ }^{4}$.

É a partir do Programa Saúde da Família (PSF), na década de 1990, um modelo para reorientar a prática assistencial, consistente com os princípios da universalidade, integralidade e equidade, que se começa a preencher o que Viana e Dal Poz denominaram de "vazio programático" desde a criação do SUS5. O PACS em 1991 e o PSF em 1994, ainda que tenham sido concebidos originalmente como programas focalizados, dirigidos a grupos da população sem acesso e com escopo seletivo de ações, apresentaram inovações para a reorientação do modelo assistencial no SUS, aliando a prática do cuidado individual com a abordagem populacional na perspectiva da vigilância em saúde com territorialização $0^{2,4,6}$. Ao longo do tempo, elementos presentes em diversos modelos, como as ações programáticas a grupos prioritários, reorganização do atendimento à demanda espontânea com acolhimento centrado no usuário e apoio matricial foram incorporados ao PSF, que passa assim a ser denominado de Estratégia de Saúde da Família (ESF) ${ }^{7,8}$.

A partir dos anos 2000, os atributos da APS sintetizados por Starfield9 (primeiro contato, abrangência/integralidade, longitudinalidade/vínculo e coordenação, orientação familiar, orientação comunitária e competência cultural) passaram a ser difundidos em publicações no país e foram incorporados à Política Nacional de Atenção Básica (PNAB), em 2006 ${ }^{4}$. É também a partir do início dos anos 2000 que esforços para avaliar os serviços de atenção básica passam a ser induzidos pelo Ministério da Saúde (MS) por meio de iniciativas de institucionalização da avaliação da APS no SUS ${ }^{10}$.

Em 2011, a PNAB passou pela primeira revisão, que alterou a concepção de APS e incorporou ações desenvolvidas por novos dispositivos, como o Núcleo de Apoio à Saúde da Família (NASF), criado em 2008, na ótica da interprofissionalidade, matriciamento, educação permanente, compartilhamento da responsabilidade clínica, para uma maior resolutividade do cuidado, e o financiamento de modalidades diferenciadas de equipes de SF. Na mesma década, destacaram-se políticas para a melhoria da infraestrutura física das UBS (Requalifica), do acesso e da qualidade (PMAQ-AB) e para a provisão de médicos (PMM), todos problemas históricos para a sustentabilidade e a expansão da APS.

Vale ressaltar, que a partir de 2017, conflitos políticos cada vez mais intensos, que se expressam tanto em ataques à democracia quanto em retrocessos nos avanços obtidos na implementação e expansão de um modelo assistencial de APS com orientação comunitária, se materializam na última revisão da PNAB e em diversas políticas subsequentes relacionadas à $\mathrm{APS}^{11,12}$.

É de se esperar que estes avanços e retrocessos repercutam no conteúdo das publicações na C\&SC, afinal uma das características marcantes da Saúde Coletiva é ser um campo que articula conhecimento e prática. Ademais, diferentemente de outros países, um dos traços da APS brasileira é sua estreita interface com a Saúde Coletiva, com importante abordagem territorializada e a presença de equipes multiprofissionais. Frente a este cenário, o objetivo do presente artigo é analisar a trajetória da produção do conhecimento sobre APS divulgada nos primeiros 25 anos na 
Foi realizada uma revisão narrativa da produção sobre APS veiculada na revista C\&SC no período de 1996 a 2019. A busca dos artigos se deu através da plataforma SciELO, que disponibiliza todos que foram publicados no período. Inicialmente, foram incluídos os artigos que continham as seguintes expressões em qualquer um dos índices de busca: "Atenção Primária", "Atenção Básica", "Saúde da Família", "Agente Comunitário de Saúde", "Estratégia Saúde da Família", "Programa de Saúde da Família". Na sequência, ampliou-se a seleção com a inclusão de artigos com as seguintes palavras chave: "Acolhimento", "Atenção Domiciliar", "Cuidados Primários de Saúde", "Programa Mais Médicos", "PMAQ", "Serviços básicos de Saúde". O último passo foi acessar todos os números temáticos que versaram sobre APS, identificando possíveis perdas das estratégias anteriores. Estes números foram identificados no sítio eletrônico da C\&SC, que disponibiliza todos os números publicados.

A busca inicial identificou 367 artigos. Cada um dos resumos foi lido, em separado, por duas pesquisadoras, que estudam APS há pelo menos 20 anos. Foram selecionados os artigos que abordavam tema pertinente ao escopo da APS e, quando houve divergência, uma terceira pesquisadora fez nova avaliação. Neste processo foram excluídos 72 artigos e os 295 incluídos foram analisados e classificados.

O tema e o subtema abordados, os métodos utilizados, o tipo de artigo, a escala de análise, as parcerias institucionais estabelecidas, o idioma de publicação e o perfil da autoria foram analisados para cada um dos artigos selecionados. Foi elaborado um banco em Excel com os principais dados bibliográficos de todos os artigos incluídos. Esta planilha foi exportada e analisada no programa IBM SPSS Statistics versão 23.

Os principais temas abordados nos artigos foram agrupados nas seguintes categorias: análise da política; financiamento, custos e aplicação de recursos; modelo assistencial; articulação da APS nas redes de atenção à saúde; gestão em saúde; processo de trabalho; gestão do trabalho; formação e educação permanente; acesso, acessibilidade,

cobertura e utilização de serviços; desempenho ou efetividade da APS; perfil de usuários; percepção dos processos de adoecimento e cuidado; promoção da saúde; família; educação em saúde; reforma de outros sistemas de saúde. As definições adotadas são apresentadas no Quadro 1.

Foram, também, identificados os "subtemas" dos artigos, considerando-se como tal os enfoques específicos na abordagem do tema, notadamente em relação a agravos, área programática, categoria profissional ou política. Por exemplo, um estudo sobre acessibilidade dos usuários no contexto do Programa Mais Médicos (PMM) foi classificado no tema "acesso, acessibilidade, cobertura e utilização de serviços" e no subtema como "Programa Mais Médicos"; um artigo que tratava do processo de trabalho dos profissionais de Saúde Bucal foi classificado quanto ao tema em "processo de trabalho", com subtema "Saúde Bucal". Todos os artigos foram classificados quanto ao tema, mas apenas aqueles que destacavam, em seus resumos, algum foco específico foram classificados quanto ao subtema.

Para os aspectos metodológicos, a primeira categorização relacionou-se ao tipo de abordagem - quantitativa ou qualitativa. Em um segundo momento, os 295 artigos foram classificados em empíricos, com utilização de dados primários ou secundários; ou teórico-conceituais, o que correspondia a um conjunto de produções - ensaios, debates, opinião e revisões - que se caracterizaram pela manifestação de juízos de valor ou pontos de vista sobre a APS, ainda que, em alguns casos, tenham sido utilizadas evidências empíricas. Em uma terceira etapa, apenas para os artigos empíricos $(n=232)$ foi identificada a abrangência do estudo - local, municipal/distrital, regional, estadual, grandes regiões/nacional, internacional - e os sujeitos envolvidos na produção dos dados - usuários, profissionais, gestores/gerentes, conselhos de saúde/participação social, outros e não se aplica (artigos em base a dados secundários e documentos).

Para a caracterização da autoria e parcerias institucionais, foram consideradas a instituição e o país do primeiro autor, o número total de autores e o número de instituições envolvidas e se incluíam instituições localizadas em mais de um estado da federação e/ou país. Os idiomas de veiculação também foram relacionados.

$\mathrm{O}$ total de artigos elencados foi comparado ao total de publicados na Revista C\&SC, recuperados manualmente na página da revista no SciELO, considerando-se todos os artigos publicados em todas seções da revista. 
Quadro 1. Detalhamento dos critérios de classificação dos artigos segundo temas, Revista Ciência \& Saúde Coletiva, 1996-2019.

\begin{tabular}{|c|c|}
\hline Tema & Detalhamento \\
\hline Modelo assistencial & $\begin{array}{l}\text { Abordagem de aspectos relacionados aos arranjos tecnológicos para a oferta do } \\
\text { cuidado na APS; no estabelecimento das relações entre serviços; na articulação da APS } \\
\text { com outros setores e apoio matricial. Foram incluídas também análise dos atributos } \\
\text { da APS, como integralidade, longitudinalidade e orientação familiar e comunitária. }\end{array}$ \\
\hline $\begin{array}{l}\text { Desempenho ou } \\
\text { efetividade da APS }\end{array}$ & $\begin{array}{l}\text { Análise de indicadores de processo ou resultado, incluindo satisfação dos } \\
\text { usuários, para avaliar o grau de implantação das ações da APS ou a qualidade do } \\
\text { desempenho do trabalho das equipes e/ou sua efetividade. }\end{array}$ \\
\hline Processo de trabalho & $\begin{array}{l}\text { Características das práticas de saúde, sua organização e as atribuições e o papel de } \\
\text { profissionais que atuam na APS. }\end{array}$ \\
\hline Gestão em saúde & $\begin{array}{l}\text { Abordagem da capacidade de governo, perfil, competências e práticas dos gestores } \\
\text { de saúde e uso de tecnologias de gestão, incluindo participação social. }\end{array}$ \\
\hline $\begin{array}{l}\text { Formação e Educação } \\
\text { Permanente }\end{array}$ & $\begin{array}{l}\text { Questões relacionadas à formação e educação permanente para profissionais das } \\
\text { equipes, incluindo avaliação de perfil e análise de competências, e apreciação dos } \\
\text { cursos de especialização e mestrado profissional. }\end{array}$ \\
\hline Análise da política & $\begin{array}{l}\text { Análise do processo de formulação e/ou implementação da política de APS/ } \\
\text { Atenção Básica, no seu conjunto, ou de um de seus componentes. }\end{array}$ \\
\hline $\begin{array}{l}\text { Acesso, acessibilidade, } \\
\text { cobertura e utilização de } \\
\text { serviços }\end{array}$ & $\begin{array}{l}\text { Avaliação do acesso, acessibilidade, cobertura e utilização de serviços, seja na } \\
\text { perspectiva de usuários e/ou profissionais e gestores, ou através de indicadores de } \\
\text { saúde. }\end{array}$ \\
\hline $\begin{array}{l}\text { Reforma de outros } \\
\text { sistemas de saúde }\end{array}$ & Análise de reformas de sistemas de saúde com foco na APS em outros países. \\
\hline $\begin{array}{l}\text { Articulação da APS nas } \\
\text { redes de atenção à saúde }\end{array}$ & $\begin{array}{l}\text { Articulação da APS com outros níveis do sistema de serviços de saúde, linhas de } \\
\text { cuidado e construção de redes de atenção. }\end{array}$ \\
\hline $\begin{array}{l}\text { Percepção dos processos } \\
\text { de adoecimento e cuidado }\end{array}$ & $\begin{array}{l}\text { Percepção dos profissionais de saúde e usuários sobre os processos de saúde- } \\
\text { doença e cuidado e sobre as representações sociais no processo saúde/doença. }\end{array}$ \\
\hline Perfil de usuários & Caracterização do perfil de usuários da APS. \\
\hline Promoção da saúde & $\begin{array}{l}\text { Abordagem de ações, propostas e programas relacionados à promoção da saúde no } \\
\text { âmbito da APS. }\end{array}$ \\
\hline Gestão do trabalho & $\begin{array}{l}\text { Formas de gestão do processo de trabalho, divisão de trabalho e comunicação, } \\
\text { política de inserção e remuneração e rotatividade dos profissionais da APS e ações } \\
\text { de vigilância à saúde do trabalhador em APS }\end{array}$ \\
\hline $\begin{array}{l}\text { Financiamento, custos e } \\
\text { aplicação de recursos }\end{array}$ & $\begin{array}{l}\text { Financiamento da APS, custos das ações e serviços, incentivos financeiro e } \\
\text { aplicação de recursos para ações de saúde }\end{array}$ \\
\hline Educação em Saúde & $\begin{array}{l}\text { Analise de práticas educativas, incluindo as representações dos profissionais de } \\
\text { saúde sobre essas práticas. }\end{array}$ \\
\hline Família & $\begin{array}{l}\text { Concepções e reflexões sobre a família e discutindo instrumentos para sua } \\
\text { abordagem. }\end{array}$ \\
\hline
\end{tabular}

\section{Resultados}

Foram analisados os 295 artigos que se debruçaram sobre a APS na Revista C\&SC, o que correspondeu a 5,9\% do total de publicações do periódico entre 1996 e 2019. O número absoluto variou no decorrer dos anos, mas apesar da variação observa-se uma linha de tendência crescente da produção sobre APS na C\&SC (Figura 1). Esta variação, em parte, decorre da publicação de oito números temáticos relacionados à APS, nestes primeiros 25 anos da C\&SC, com 107 artigos publicados nos fascículos temáticos. Vale ressaltar que o primeiro artigo sobre o tema foi publicado apenas em 2002.

Os números específicos concentraram 36,3\% $(\mathrm{n}=107)$ de toda a produção sobre a APS. Os temas abordados foram: a avaliação como estratégia de mudança; a multiprofissionalidade no então Programa de Saúde da Família; a Atenção Básica no cuidado de doenças e agravos; a expansão e os desafios da Saúde da Família; a Atenção 


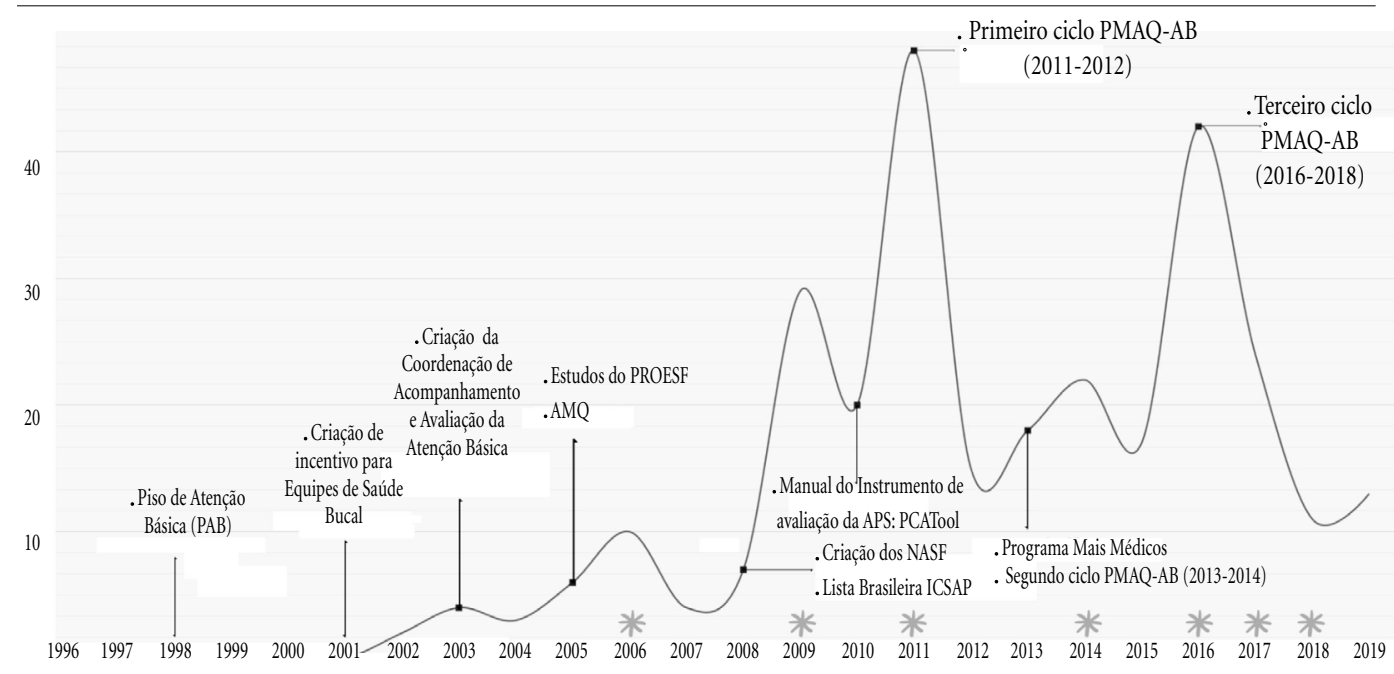

Figura 1. Artigos de APS publicados na C\&SC e principais marcos avaliativos, 1996-2019.

*Anos com números temáticos versando sobre APS.

Básica e a Saúde da Família; a Reforma da APS no município do Rio de Janeiro; o Programa Mais Médicos; e, por último, uma análise comparada da APS nas cidades de Lisboa e do Rio de Janeiro.

A média do número de autores por artigo foi de 3,9, com mediana de 3 e desvio padrão de 2,1 . Quanto ao idioma, apenas 10 artigos (3,4\%) foram publicados exclusivamente em outra língua que não o português. Observou-se o crescimento da publicação em dois idiomas, especialmente nos últimos cinco anos, sendo que 29,8\% das publicações também foram publicadas em inglês e apenas seis artigos em espanhol.

Considerando-se as instituições dos primeiros autores, o periódico veiculou, fundamentalmente, autores brasileiros (93,6\%), seguidos de portugueses $(3,7 \%)$, sendo a maioria mulheres $(63,7 \%)$. Entre os brasileiros, os autores provinham predominantemente de instituições do Rio de Janeiro (18,0\%), São Paulo (17,6\%), Distrito Federal (9,5\%), Minas Gerais (9,2\%), Rio Grande do Sul $(8,5 \%)$. Não foram identificados primeiros autores de instituições do Acre, Roraima, Rondônia, Amapá, Sergipe, e apenas dois artigos com vinculações no estado do Amazonas e um do Pará.

Embora a maioria dos estudos tenha sido realizada por pesquisadores de mais de uma instituição (59,0\%), em apenas $24,1 \%$ destes, esta colaboração abarcava diferentes estados da federação (Tabela 1).
A maior parte dos estudos utilizou abordagem qualitativa $(58,0 \%)$. O predomínio de estudos foi empírico $(78,6 \%)$, embora percentual importante tenha sido caracterizado como artigos teórico-conceituais que incluiu ensaios, debates, revisão e artigos de opinião. A maioria dos artigos empíricos $(68,1 \%)$ foi de âmbito local e municipal/distrital e poucos foram de abrangência nacional $(11,2 \%)$, em geral, decorrentes de processos avaliativos induzidos pelo Ministério da Saúde como o Proesf e Autoavaliação para a Melhoria do Acesso e da Qualidade da Atenção Básica (AMAQ), além de estudos que utilizaram a abordagem das Internações por Condições Sensíveis à Atenção Primária (ICSAP) e do Primary Care Assessment Tool (PCATool) (Tabela 1).

Quanto à população estudada, foram mais frequentes os estudos realizados com profissionais de saúde, seguidos daqueles com usuários e gestores. Abordagens com mais de uma categoria foram mais raras (Tabela 1). Apenas três artigos focaram nos conselhos de saúde. Ademais, quase um quarto dos estudos empíricos utilizou dados secundários e/ou documentos como fonte de dados, agrupados como NSA (Tabela 1).

Quanto à classificação temática, três temas predominaram, correspondendo, juntos, a mais de 50\% dos estudos: modelo assistencial (18,3\%), desempenho ou efetividade da APS (17,0\%) e processo de trabalho (15,3\%) (Tabela 2 ). Se agregarmos aos três anteriores os temas de "Acesso, 
Tabela 1. Características dos artigos de APS publicados na C\&SC de 1996 a 2019.

\begin{tabular}{llrr}
\hline \multicolumn{1}{c}{ Características } & & No. & $\%$ \\
\hline Abordagem $(\mathrm{n}=295)$ & Qualitativa & 171 & 58,0 \\
& Quantitativa & 106 & 35,9 \\
Tipo de Estudo $(\mathrm{n}=295)$ & Quali/quantitativa & 18 & 6,1 \\
& Empírico & 232 & 78,6 \\
Abrangência dos Estudos Empíricos $(\mathrm{n}=232)$ & Ensaio, Debate e Opinião & 55 & 18,6 \\
& Revisão & 8 & 2,7 \\
& Local (UBS) & 47 & 20,3 \\
& Municípios/Distritos & 111 & 47,8 \\
Sujeitos ( $\mathrm{n}=232)$ & Região & 15 & 6,5 \\
& Estado & 19 & 8,2 \\
& Grandes Regiões/Nacional & 26 & 11,2 \\
& Internacional & 15 & 6,0 \\
Instituições envolvidas $(\mathrm{N}=295)$ & Profissionais & 77 & 33,2 \\
Unidades da Federação & Usuários & 40 & 17,2 \\
& Gestores & 22 & 9,5 \\
& Profissionais e Gestores & 15 & 6,5 \\
& Usuário, profissional e gestor & 10 & 4,3 \\
& Usuário e Profissional & 8 & 3,4 \\
& Outros & 4 & 1,8 \\
& NSA & 56 & 24,1 \\
& Uma & 121 & 41,0 \\
& Duas ou mais & 174 & 59,0 \\
& Uma & 220 & 74.6 \\
& Duas ou mais & 71 & 24,1 \\
& NSA & 1,3 \\
\hline
\end{tabular}

Fonte: Elaboração das autoras.

acessibilidade, cobertura e utilização de serviços" $(4,8 \%)$ e "Articulação da APS nas redes de atenção" $(3,4 \%)$, podemos perceber que o foco dos pesquisadores em quase $60 \%$ dos artigos sobre APS publicados no periódico foram aspectos relacionados à direcionalidade e efetividade das mudanças da APS em relação ao processo de construção do SUS e à Reforma Sanitária Brasileira. Os 40\% restantes dos artigos abordavam diversos temas, sendo que, isoladamente, nenhum alcançou percentual igual ou superior a $10 \%$ : "Gestão em saúde" (9,2\%), "Formação e Educação Permanente" (7,5\%), "Análise da política brasileira de APS" (5,8\%), "Reforma de outros sistemas de saúde" (4,1\%), "Articulação da APS nas redes de atenção à saúde" (3,4\%), "Percepção do adoecimento e cuidado" (3,7\%) e outros, que não alcançaram 3\% dos artigos analisados (Tabela 2). Em relação à reforma de outros sistemas de saúde no mundo, foram publicados artigos que analisavam a APS em Portugal, Alemanha, Chi- le, Paraguai, Uruguai, Argentina e Espanha e no conjunto da América Latina.

Comparando-se os artigos empíricos ou de revisão com o grupo de artigos que agregavam os ensaios, debates e artigos de opinião, "Modelo assistencial" e "Processo de trabalho" permaneceram como temas privilegiados em ambos os grupos. A imensa maioria dos artigos que abordaram o "Desempenho ou efetividade da APS" era do grupo de artigos empíricos ou de revisão, enquanto a "Análise da política" de um modo geral foi abordada em ensaios, debates ou artigos de opinião.

Para 227 artigos foi possível identificar um subtema específico, os quais foram agrupados na Tabela 3 em função de certas similaridades no tocante ao enfoque. Observamos que cerca de $30 \%$ dos artigos tiveram como foco alguma área programática da APS, com destaque para a Saúde Bucal $(7,9 \%$ do total e mais de $25 \%$ dos artigos desse subgrupo). Um outro subgrupo que 
Tabela 2. Classificação temática dos artigos de APS publicados na C\&SC, 1996 a 2019.

\begin{tabular}{lrrrrrr}
\hline \multicolumn{1}{c}{ Tema } & \multicolumn{2}{c}{ Todos } & \multicolumn{2}{c}{$\begin{array}{c}\text { Empíricos e } \\
\text { Revisões }\end{array}$} & $\begin{array}{c}\text { Ensaios, debates e } \\
\text { de opinião }\end{array}$ \\
\hline & \multicolumn{1}{c}{$\mathbf{N}$} & \multicolumn{1}{c}{$\%$} & $\mathbf{N}$ & \% & N & \multicolumn{1}{c}{$\%$} \\
\hline Modelo assistencial & 54 & 18,3 & 40 & 16,7 & 14 & 25,5 \\
Desempenho ou efetividade da APS & 50 & 17,0 & 49 & 20,4 & 1 & 1,8 \\
Processo de trabalho & 45 & 15,3 & 36 & 15,0 & 9 & 16,4 \\
Gestão em Saúde & 27 & 9,2 & 22 & 9,2 & 5 & 9,1 \\
Formação e Educação Permanente & 22 & 7,5 & 20 & 8,3 & 2 & 3,6 \\
Análise da política & 17 & 5,8 & 5 & 2,1 & 12 & 21,8 \\
Acesso, cobertura e utilização & 14 & 4,8 & 14 & 5,8 & 0 & 0,0 \\
Reforma de outros sistemas de saúde & 12 & 4,1 & 8 & 3,3 & 4 & 7,3 \\
APS nas Redes e Regiões & 10 & 3,4 & 8 & 3,3 & 2 & 3,6 \\
Percepção do adoecimento e cuidado & 9 & 3,1 & 9 & 3,8 & 0 & 0,0 \\
Perfil de usuários & 8 & 2,7 & 7 & 2,9 & 1 & 1,8 \\
Promoção da saúde & 8 & 2,7 & 6 & 2,5 & 2 & 3,6 \\
Gestão do trabalho & 7 & 2,4 & 7 & 2,9 & 0 & 0,0 \\
Financiamento, custos e aplicação & 6 & 2,0 & 5 & 2,1 & 1 & 1,8 \\
Educação em Saúde & 4 & 1,4 & 4 & 1,7 & 0 & 0,0 \\
Família & 2 & 0,7 & 0 & 0,0 & 2 & 3,6 \\
Total & 295 & 100,0 & 240 & 100,0 & 55 & 100,0 \\
\hline
\end{tabular}

Fonte: Elaboração das autoras.

ganhou expressão nessa análise, com 16,7\%, é o que abordou aspectos relativos aos profissionais da equipe, como por exemplo, práticas ou processos de educação permanente, sendo que em metade dos casos o profissional em questão foi o ACS. Foi possível identificar como subtemas relacionados ao modelo de atenção à saúde, como vigilância da saúde e participação social, apoio matricial e atributos da APS, em cerca de $10 \%$ dos artigos. Um número expressivo de artigos, correspondendo a quase $10 \%$, se debruçou sobre agravos e problemas de saúde, especialmente as doenças crônicas (3,5\% do total de artigos). Finalmente, vale registrar o elevado percentual que tratou do Programa Mais Médicos (7,9\%), que foi objeto de um suplemento especial. Os demais subtemas foram bastante pulverizados, com percentuais em torno ou abaixo de 5\%, indicando o abrangente leque de aspectos investigados da APS.

\section{Discussão e considerações}

O presente artigo teve como objetivo identificar a produção científica sobre APS publicada na C\&SC. Desta forma, embora dialogue com a produção do campo da Saúde Coletiva brasilei- ra sobre o tema, seus resultados aqui apresentados são circunscritos aos artigos publicados na C\&SC. O volume encontrado vai ao encontro dos resultados obtidos por Medina et al. ${ }^{13}$, que identificaram uma importante produção sobre APS brasileira, chegando a mais de 860 artigos entre 1980 e 2016. Embora os critérios de seleção adotados tenham sido diferentes dos que foram aqui utilizados, as autoras identificaram que a C\&SC foi o segundo periódico com maior veiculação da produção, perdendo apenas para o Cadernos de Saúde Pública, em um total de 153 periódicos. Neste cenário de grande dispersão na veiculação do conhecimento sobre APS, o número de artigos aqui encontrados corrobora que a $\mathrm{C} \& S C$ foi e continua sendo um periódico central para a construção do conhecimento sobre a APS brasileira, no contexto do SUS.

O primeiro artigo sobre APS na C\&SC foi publicado apenas em 2002, sete anos após o início do periódico e cerca de uma década após o início da experiência do PACS/PSF, coincidindo com o incremento da cobertura pela ESF no Brasil. Este hiato entre a prática e a veiculação da produção científica, especialmente a produção com base empírica, não é exclusivo da C\&SC, nem da produção brasileira ${ }^{13,14}$. Hirschhorn et al. ${ }^{15}$ reforçam a necessidade premente de se produzir, 
Tabela 3. Distribuição dos artigos publicados na C\&SC por subtema de 1996 a 2019.

\begin{tabular}{|c|c|c|}
\hline Subtema & $\mathbf{N}$ & $\%$ \\
\hline Profissionais de saúde & 38 & 16,7 \\
\hline Agentes Comunitários de Saúde & 19 & 8,4 \\
\hline Outros profissionais & 19 & 8,4 \\
\hline Programa Mais Médicos & 18 & 7,9 \\
\hline Aspectos dos processos de trabalho & 9 & 4,0 \\
\hline Equipe interdisciplinar e trabalho em equipe & 6 & 2,6 \\
\hline Outros (práticas assistenciais, tecnologias leves e rotatividade dos profissionais) & 4 & 1,8 \\
\hline Especialização e Mestrado & 3 & 1,3 \\
\hline Contratualização e outras formas de gestão do trabalho & 5 & 2,2 \\
\hline Programas de incentivos & 5 & 2,2 \\
\hline Agravos e problemas de saúde & 22 & 9,7 \\
\hline Doenças crônicas & 8 & 3,5 \\
\hline ICSAP & 6 & 2,6 \\
\hline $\begin{array}{l}\text { Outros (Controle de tabagismo, dengue, tuberculose, pessoas com deficiência, } \\
\text { Síndrome de Down, violência) }\end{array}$ & 8 & 3,5 \\
\hline Áreas programáticas & 68 & 30,0 \\
\hline Saúde Bucal & 18 & 7,9 \\
\hline Saúde da Criança e Materno-infantil & 12 & 5,3 \\
\hline Saúde do Homem & 7 & 3,1 \\
\hline Saúde do Idoso & 7 & 3,1 \\
\hline Assistência Farmacêutica & 6 & 2,6 \\
\hline Práticas Integrativas e Complementares & 5 & 2,2 \\
\hline Outros & 13 & 5,7 \\
\hline Enfoques do modelo assistencial & 24 & 10,6 \\
\hline Vigilância da Saúde e participação social & 8 & 3,5 \\
\hline Apoio matricial & 7 & 3,1 \\
\hline Outros & 9 & 4,0 \\
\hline Avaliação, monitoramento e Sistemas e tecnologias de Informação & 10 & 4,4 \\
\hline Saúde internacional & 12 & 5,3 \\
\hline Portugal & 5 & 2,2 \\
\hline Outros países & 7 & 3,1 \\
\hline Outros & 12 & 5,3 \\
\hline Total & 227 & 100 \\
\hline
\end{tabular}

Fonte: Elaboração das autoras.

com agilidade, pesquisas e novas evidências para o fortalecimento da APS nos sistemas de saúde. Desde a publicação do primeiro artigo, nota-se que sua produção se expande, acompanhando a prioridade política conferida à Saúde da Família, com todos os desafios colocados neste processo ${ }^{16}$.

O número de artigos publicados anualmente foi bastante variável, o que pode refletir tanto investimento nos processos avaliativos implementados pelo Ministério da Saúde, quanto a publicação de números temáticos. Apesar desta variação, pode-se afirmar, sem dúvida, que a APS é um tema importante e com espaço crescente na C\&SC.
Se no geral, observa-se um crescimento da produção sobre APS na C\&SC, esta veiculação ainda reflete, por um lado, uma grande concentração de primeiros autores de estados mais ricos da federação e, por outro, a ausência de autores de diversos estados, sendo que a quase ausência de primeiros autores da região Norte é significativa da desigual produção de conhecimento. De certa forma, este quadro também espelha a distribuição dos cursos de mestrado e doutorado da Saúde Coletiva pelo país.

A produção sobre APS na C\&SC remete-se fundamentalmente às experiências locais, por meio de abordagem qualitativa, veiculadas em 
língua portuguesa. Pode-se observar um "diálogo para dentro", talvez influenciado, entre outros aspectos, pelo modelo de descentralização do sistema de saúde brasileiro, cujas transferências financeiras do nível central para os municípios, assim como a atribuição de responsabilidades pela implementação ao ente local, produziu um mosaico de experiências em APS, retratadas pela produção acadêmica na área. Embora esta seja uma característica da rica e diversa APS brasileira, permanece o desafio de tornar a produção científica mais reconhecida e debatida internacionalmente; certamente a veiculação em mais de um idioma contribuirá neste sentido.

Outro aspecto que não pode ser esquecido, quando da análise da produção sobre APS, é a forte influência das políticas federais de institucionalização do monitoramento e avaliação da APS pela gestão federal. Um ponto de inflexão foi a criação, em 2003, da Coordenação de Acompanhamento e Avaliação da Atenção Básica no DAB/SAS/MS ${ }^{17}$. Desenvolveram-se, também, iniciativas do Departamento de Ciência e Tecnologia (Decit) da Secretaria de Ciência, Tecnologia e Insumos Estratégicos / MS e editais conjuntos com o Conselho Nacional de Desenvolvimento Científico e Tecnológico (CNPQ), além do apoio às agências estaduais de fomento de pesquisa no Programa Pesquisa para o SUS, que podem justificar o aumento da veiculação da produção sobre o tema na C\&SC.

A estratégia do MS de incluir as universidades como parceiras nos processos avaliativos, sem dúvida, contribuiu para o crescimento da produção científica sobre o tema. Esta estratégia, inicialmente com financiamento para a realização de pesquisas em avaliação com solicitações esparsas a instituições acadêmicas, evoluiu, em 2005, para o Projeto de Expansão e Consolidação da Estratégia Saúde da Família (PROESF), que promoveu um conjunto de estudos avaliativos de Linha de Base, englobando 227 municípios com população acima de 100.000 habitantes, o que impulsionou a pesquisa avaliativa em atenção primária, especialmente com a utilização de desenho transversal, combinação de métodos e estudo de casos por amostra intencional, e abordagem a gestores, profissionais e usuários. Estes estudos marcaram presença na C\&SC ${ }^{18-20}$.

Por outro lado, observa-se que outro grande movimento avaliativo no cenário da APS, o Programa de Melhoria do Acesso e Qualidade da Atenção Básica (PMAQ-AB) não se refletiu de forma expressiva na produção sobre APS na revista C\&SC. Os estudos provenientes do PMA-
Q-AB veiculados nos diversos periódicos, no geral, se apoiaram em desenhos transversais junto a profissionais e usuários da APS, combinando métodos de abordagem mista (quali-quanti), e técnicas diversas de pesquisa que permitiam valorizar a percepção de seus executores e dos cidadãos beneficiários quanto aos problemas da atenção em termos de estrutura, processos e satisfação das necessidades de saúde ${ }^{21}$. Já na C\&SC, os artigos que se apoiam nos bancos de dados do PMAQ-AB dos dois primeiros ciclos de avaliação trabalharam novas hipóteses quando os associam a outros programas, como o PMM ou a eficácia da formação em saúde da família para a integralidade da atenção na $\mathrm{ESF}^{22,23}$.

Em 2013, o Programa Mais Médicos produziu uma inflexão na política nacional de atenção básica, trazendo à luz as imensas dificuldades de prestação de serviços em áreas remotas e de maior vulnerabilidade ${ }^{24}$. Tal política, em curto período de tempo, gerou um conjunto expressivo de publicações, sendo o número especial da C\&SC sobre o PMM, produzido em 2016, considerado o principal veículo para a divulgação de artigos de opinião e debate sobre o PMM, assim como artigos empíricos com resultados de pesquisa $^{25}$.

O conjunto da produção da C\&SC ainda está focada na dimensão nacional, com diversos estudos empíricos que se debruçam na análise da implementação de políticas estruturantes voltadas para o fortalecimento da APS como o Proesf, PMAQ e PMM e/ou estudos teóricos, de revisão ou ensaios, que tentam captar a evolução do processo de estruturação da APS no país. Contudo, análises com escala nacional, com base nos dados produzidos por estes processos, é pequena, especialmente quando se toma como parâmetro a ampla disponibilização do conjunto de dados relativos à situação da APS no Brasil ${ }^{26}$. Esta baixa difusão destes estudos contrasta com o processo de estruturação da APS no Brasil, que nas últimas décadas tem consolidado um modelo assistencial de abrangência nacional, com o estabelecimento de práticas, normativas, inovações e reflexões advindas das distintas práticas de gestão de âmbito nacional, com forte diálogo entre os níveis de governo, o que configura a rica experiência brasileira em APS em termos de renovação dos modelos de gestão e de atenção em razão do processo de descentralização da saúde.

A presença significativa de artigos empíricos de abrangência municipal ou local, próximo de $70 \%$ dos estudos, foi constatado também em outras revisões ${ }^{13,27-29}$. Este resultado associa-se por 
um lado ao processo de protagonismo dos municípios em experiências inovadoras, que passam a se constituir com frequência como objetos de estudo, mas mostra também descompasso entre a produção científica em APS e o amplo programa nacional de APS - uma maioria de estudos qualitativos, centrados em poucos grupos de pesquisa, com pouca elaboração compartilhada com outros grupos.

Quanto aos temas, o predomínio de análises sobre o "modelo assistencial, desempenho ou efetividade da APS" e "processo de trabalho" revela a preocupação dos pesquisadores em analisar as mudanças esperadas no tocante ao processo de implementação do modelo brasileiro de APS, na reorientação das práticas assistenciais e sua lógica organizacional, bem como os resultados de tais mudanças em termos de indicadores de saúde populacional. No primeiro caso, os resultados pretendem contribuir com o conhecimento dos aspectos relacionados aos arranjos tecnológicos da organização do cuidado em APS, incluindo dispositivos como o apoio matricial às equipes, $\mathrm{e}$ destacando a investigação dos principais atributos da APS, como integralidade, longitudinalidade e orientação familiar e comunitária.

De fato, o debate em torno do modelo de atenção à saúde é central no processo de construção do SUS, caudatário de um movimento de reformas de sistemas de saúde no contexto internacional, e expressa disputas entre o que veio a ser designado por modelo biomédico versus modelos alternativos que deslocam a centralidade do objeto e da organização dos serviços do binômio doença-hospital para o indivíduo-família-comunidade e território onde se promove a saúde e se produz o cuidado ${ }^{30,31}$. A política de APS embora destacada enquanto temática específica, apresenta certa transversalidade e interface com outros temas apresentados nos artigos como "processo de trabalho", "articulação da APS nas redes de atenção à saúde" e é tratada em diversos estudos que têm como foco a análise global da política.

A análise da efetividade da APS na organização dos serviços e na saúde da população é um tema que vem ganhando espaço na literatura nacional e internacional ${ }^{26,32,33}$, uma vez que a APS se constitui em cenário privilegiado para a indução de mudanças significativas nos padrões de adoecimento dos indivíduos e populações. Isto porque ao imprimir uma nova dinâmica organizacional nas unidades de saúde, a APS é capaz de promover a incorporação de novas práticas de promoção da saúde e prevenção de agravos, além de ampliar a acessibilidade às ações e aos serviços tanto de APS quanto de outros níveis de complexidade do sistema de saúde. Em relação ao terceiro tema mais abordado, processo de trabalho, os autores aprofundaram as discussões sobre as práticas dos profissionais e suas atribuições, incluída a reflexão sobre o papel de diversas categorias de saúde, em especial do agente comunitário de saúde. O destaque do ACS revela a sua relevância na construção do modelo de atenção primária brasileiro, como têm destacado diversos autores ${ }^{34-36}$.

Os artigos que abordaram a gestão da saúde, destacando o perfil, as competências e as práticas dos gestores, incluindo o uso de tecnologias, e aqueles que discorreram sobre as diversas iniciativas de formação e educação permanente dos profissionais de saúde, demonstram a preocupação com a necessária qualificação da APS para o cumprimento de suas funções essenciais.

Vale destacar a pouca presença de outras temáticas relevantes, como articulação da APS na rede de atenção, gestão do trabalho, financiamento, promoção da saúde, educação em saúde e reflexões sobre a família - lacunas importantes do conhecimento que merecem ser aprofundadas e que representam obstáculos a serem superados pela APS no Brasil.

Sem dúvida, as páginas publicadas nos primeiros 25 anos da C\&SC evidenciaram os debates e contribuíram para a reflexão e a divulgação da rica experiência da APS brasileira. Mantendo esta importante tradição, em 2020 foi publicado um número especial sobre os 40 anos de Alma Ata e convidamos os leitores a visitá- $l^{37}$. O conjunto da produção sobre APS nestes 25 anos aporta reflexões centrais para a consolidação do projeto de um sistema de saúde equânime e integral no Brasil, o nosso SUS. Com certeza, as páginas futuras da C\&SC continuarão a veicular estas experiências e debates. 


\section{Colaboradores}

A Bousquat, MG Medina, MHM Mendonça, PF Almeida, R Aquino, AF Santos e L Giovanella participaram igualmente de todas as etapas de elaboração do artigo.

\section{Referências}

1. Minayo MCS, Gomes R. Cien Saude Colet no contexto nacional e internacional da divulgação científica. Cien Saude Colet 2015; 20(7):2013-2022.

2. Paim J. Atenção primária à saúde: uma receita para todas as estações? Saúde em Debate 2012; 36(94):343347.

3. Paim JS. Modelos de Atenção à Saúde no Brasil. In: Giovanella L, Escorel Sarah, Lobato LVC, Noronha JC, Carvalho AI, organizadores. Politicas e Sistema de Saúde no Brasil. Rio de Janeiro: Editora Fiocruz; 2012. p. 459-492.

4. Giovanella L, Mendonça MHM. Atenção Primária à Saúde. In: Giovanella L, Escorel Sarah, Lobato LVC, Noronha JC, Carvalho AI, organizadores. Políticas e Sistema de Saúde no Brasil. Rio de Janeiro: Editora Fiocruz; 2012. p. 493-346.

5. Viana AL, Dal Poz MR. A Reforma do Sistema de Saúde no Brasil e o Programa de Saúde da Família. Physis 2005; 15(Supl.):225-264.

6. Teixeira CF. A mudança do modelo de atenção à saúde no SUS: desatando nós, criando laços. In: Teixeira CF, Solla JP, organizadores. Modelo de atenção à saúde: promoção, vigilância e saúde da família. Salvador: Edufba; 2006. p. 19-58.

7. Schraiber LB, organizador. Programação em Saúde Hoje. São Paulo: Hucitec; 1993.

8. Cunha GT, Campos GWS. Apoio Matricial e Atenção Primária em Saúde. Saude Soc 2011; 20(4):961-970.

9. Starfield B. Atenção Primária: equilíbrio entre necessidades de saúde, serviços e tecnologia. Brasília: Unesco, Ministério da Saúde (MS); 2002.

10. Almeida PF, Giovanella L. Avaliação em Atenção Básica à Saúde no Brasil: mapeamento e análise das pesquisas realizadas e/ou financiadas pelo Ministério da Saúde entre os anos de 2000 e 2006. Cad Saude Publica 2008; 24(8):1727-1742.

11. Melo EA, Mendonca MHM, Oliveira JP, Andrade GCL. Mudanças na Política Nacional de Atenção Básica: entre retrocessos e desafios. Saúde debate 2018; 42(n esp. 1):38-51.

12. Giovanella L, Bousquat A, Almeida PF, Melo EA, Medina MG, Aquino R, Mendonça MHM. Médicos pelo Brasil: caminho para a privatização da atenção primária à saúde no Sistema Único de Saúde? Cad Saude Publica 2019; 35(10):e0178619.

13. Medina MG, Aquino R, Vilasbôas ALQ, Nunes CA. A Pesquisa em Atenção Primária à Saúde no Brasil. In: Mendonça MHM, Matta G, Gondin R, Giovanella L, organizadores. Atenção Primária à Saúde no Brasil: Conceitos, Práticas e Pesquisa. Rio de Janeiro: Editora Fiocruz; 2018. p: 453-492.

14. Hummers-Pradiers E, Scheidt-Nave C, Martin H, Heinemann S, Kochen MM, Himmel W. Simply no time? Barriers to GP's participation in primary health care research. Family Practice 2008; 25(2):105-112.

15. Hirschhorn LR, Langlois EV, Bitton A, Ghaffar A. What kind of evidence do we need to strengthen primary healthcare in the 21st century? BMJ Global Health 2019; 4(Supl. 8):e001668

16. Sousa MF, Hamann EM. Programa Saúde da Família no Brasil: uma agenda incompleta? Cien Saude Colet 2009; 14(Supl. 1):1325-1335. 
17. Felisberto E. Da teoria à formulação de uma Política Nacional de Avaliação em Saúde: reabrindo o debate. Cien Saude Colet 2006; 11(3):553-563.

18. Bodstein R, Feliciano K, Hortale VA, Leal MC. Estudos de Linha de Base do Projeto de Expansão e Consolidação do Saúde da Família (ELB/Proesf): considerações sobre seu acompanhamento. Cien Saude Colet 2006, 11(3):725-731.

19. Elias PE, Ferreira CW, Alves MCG, Cohn A, Kishima V, Escrivão Junior A, Gomes A, Bousquat A. Atenção Básica em Saúde: comparação entre PSF e UBS por estrato de exclusão social no município de São Paulo. Cien Saude Colet 2006; 11(3):633-641.

20. Facchini LA, Piccini RX, Tomasi E, Thumé E, Silveira DS, Siqueira FV, Rodrigues MA. Desempenho do PSF no Sul e no Nordeste do Brasil: avaliação institucional e epidemiológica da Atenção Básica à Saúde. Cien Saude Colet 2006, 11(3):669-681.

21. Mendonça MHM, Giovanella L, Fausto MCR, Lima JG, Escorel S, Almeida PF, Pilar AA. Melhoria da Qualidade nas práticas de Atenção Primária à Saúde. In: Sá MC, Tavares MFL, De seta MH, organizadores. Organização do Cuidado e Práticas em Saúde - abordagens, pesquisa e experiências de ensino. Rio de Janeiro: Editora Fiocruz; 2018. p. 257-293.

22. Giovanella L, Mendonça MHM, Fausto MCR, Almeida PF, Bousquat A, Lima JG, Seidl H, Franco CM, Fusaro ER, Almeida SZF. A provisão emergencial de médicos pelo Programa Mais Médicos e a qualidade da estrutura das Unidades Básicas de Saúde. Cien Saude Colet 2016; 21(9):2697-2608.

23. Santos NML, Hugo FN. Formação em Saúde da Família e sua associação com processos de trabalho das Equipes de Saúde Bucal da Atenção Básica. Cien Saude Colet 2018; 23(12):4319-4329.

24. Organização Pan-Americana da Saúde (OPAS). Programa Mais Médicos no Brasil. Panorama da Produção Científica. Brasília: OPAS; 2017

25. Facchini LA, Batista SR, Silva Júnior AG, Giovanella L. O Programa Mais Médicos: análises e perspectivas. Cien Saude Colet 2016; 21(9):2652.

26. Facchini LA, Tomasi E, Dilélio AS. Qualidade da Atenção Primária à Saúde no Brasil: avanços, desafios e perspectivas. Saúde Debate 2018; 42(n. esp. 1):208-223.

27. Giovanella L, Mendonça MHM, Medina MG, Lima JG, Fausto MCR, Seidl HM, Andrade GCL, Facchini LA. Contribuições dos Estudos PMAQ-AB para a avaliação da APS no Brasil. In: Mendonça MHM, Matta G, Gondim R, Giovanella L, organizadores. Atenção Primária à Saúde no Brasil: Conceitos, Práticas e Pesquisa. Rio de Janeiro: Editora Fiocruz; 2018. p. 569610.

28. Ribeiro LA, Scatena JH. Avaliação da atenção primária à saúde no contexto brasileiro: uma análise da produção científica entre 2007 e 2017. Saúde Soc 2019; 28(2):95-110.
29. Arantes LJ, Shimizu HE, Merchán-Hamann E. Contribuições e desafios da Estratégia Saúde da Família na Atenção Primária à Saúde no Brasil: revisão da literatura. Cien Saude Colet 2016; 21(5):1499-1510.

30. Teixeira CF, Vilasbôas ALQ. Modelos de atenção à saúde no SUS: transformação, mudança ou conservação? In: Paim JS, Almeida-Filho N, organizadores. Saúde Coletiva: teoria e prática. Rio de Janeiro; Medbook: 2014. p 287-301.

31. Teixeira CF. PAIM JS, Vilasbôas ALQ. SUS, modelos assistenciais e vigilância da saúde. Informe Epidemiológico do SUS 1998; 7(2):7-28.

32. Macinko J, Starfield B, Erinosho T. The impact of primary healthcare on population health in low- and middle-income countries. J Ambul Care Manage 2009; 32(2):150-171.

33. Rede de Pesquisa em Atenção Primária à Saúde da Abrasco. Contribuição para uma agenda política estratégica para a Atenção Primária à Saúde no SUS. Saúde em Debate 2018; 42(n. esp. 1):406-430.

34. Costa SM, Araújo FF, Martins LV, Nobre LLR, Araújo FM, Rodrigues CAQ. Agente Comunitário de Saúde: elemento nuclear das ações em saúde. Cien Saude Colet 2013; 18(7):2147-2156.

35. Gomes KO, Cotta RMM, Mitre SM, Batista RS, Cherchiglia ML. O Agente

36. Comunitário de Saúde e a consolidação do Sistema Único de Saúde: reflexões contemporâneas. Physis 2010; 20(4):1143-1164.

37. Pereira IC, Oliveira MAC. O trabalho do agente comunitário na promoção da saúde: revisão integrativa da literatura. Rev Bras Enferm 2013; 66(3):412-419.

38. Pinto LP, Soranz D, Ponka D, Pisco LA, Hartz ZM. 40 anos de Alma-Ata: desafios da Atenção Primária à Saúde no Brasil e no mundo. Cien Saude Colet 2020; 25(4):1178-1178.

Artigo apresentado em 01/07/2020

Aprovado em 02/07/2020

Versão final apresentada em 04/07/2020 